\title{
Do Long Term Cancer Survivors Have Better Health-Promoting Behavior than Non-Cancer Populations?: Case-Control Study in Korea
}

\author{
Sung-Youn Chun ${ }^{1,2}$, Hyeki Park ${ }^{1,2}$, Tae Hoon Lee ${ }^{1,2}$, Eun-Cheol Park ${ }^{2,3 *}$
}

\begin{abstract}
Background: We compared the health-promoting behavior of long-term cancer survivors with those of the general population to identify necessary behavioral interventions to reduce the health risk among cancer patients. Materials and Methods: We used data from the 2007 and 2012 Korea National Health and Nutrition Examination Surveys (KNHANES IV [2007 2009] and KNHANES V [2010 2012]) on smoking status, alcohol use, physical exercise, and disease screening. We compared long-term cancer survivors with members of the general population; the controls were matched by propensity score matching. A multiple logistic regression model was used to investigate the association between cancer status and health-promoting behavior. Results: Long-term cancer survivors had a lower risk of smoking than the general population controls (OR: 0.42,95\% CI: 0.25-0.71). In addition, the long-term cancer survivors had a lower risk of alcohol use than the general population controls (OR: 0.70, 95\%CI: 0.50-0.98). However, in terms of physical exercise and disease screening, no statistically significant differences were detected (physical exercise OR: 1.01,95\% CI: 0.75-1.35; disease screening OR: 1.27, 95\% CI: 0.93-1.74). All covariates were adjusted. Conclusions: The long-term cancer survivors had a much lower risk of smoking and alcohol use than the general population controls. However, almost no differences in physical exercise and screening for cancer recurrence or secondary disease were detected between the long-term cancer survivors and general population controls. To reduce the health risks and challenges facing long-term cancer survivors, interventions to encourage physical exercise and screening for cancer recurrence and secondary disease should be implemented.
\end{abstract}

Keywords: Cancer - long-term survivors - health-promoting behavior - case-control study - Korea

Asian Pac J Cancer Prev, 16 (4), 1415-1420

\section{Introduction}

The number of long-term cancer survivors in the world is growing and will continue to grow more due to aging societies and advances in cancer treatment. While the overall cancer incidence rates from 2002-2011 in Korea increased by $3.4 \%$ per year, mortality decreased by $2.7 \%$ per year. The five-year relative survival rates of patients diagnosed in the last five years (2007-2011) improved by $25.1 \%$ compared with those from 1993-1995; thus, many people are living with cancer as a chronic disease. Although the increase in cancer survivorship is encouraging, survivors havea significantly elevated risk of recurrence and secondary cancers, comorbid chronic conditions and lingering physical symptoms, and premature mortality related to the cancer and the consequences of treatment (Ihira et al., 2014; Low et al., 2014).

On the other hand, positive health-promoting behaviors, including non-smoking status, alcohol non-use, lifestyle factors such as physical activity, and appropriate health service utilization such as screening for secondary cancer can minimize the health risks and challenges facing cancer survivors (Carmack et al., 2011; Lee and Loh, 2013).

Few studies have investigated positive healthpromoting behaviors among long-term cancer survivors (Hudson et al., 2009; Hawkins et al., 2010) and, to our knowledge, these studies were mostly based on nonAsian populations. Therefore, the differences in healthpromoting behaviors among Asian populations according to a multiple regression model as part of a case control study are unclear. The goal of this study was to examine the adherence of long-term cancer survivors in Korea to positive health-promoting behaviors. We also examined the differences in health-promoting behaviors between the general population and long-term cancer survivors. We hypothesized that health-promoting behaviors such as smoking status, alcohol use, physical activity, and disease screening would differ between the general population and long-term cancer survivors.

Thus, our goal was to determine which health-

${ }^{1}$ Department of Public Health, Graduate School, ${ }^{2}$ Institute of Health Services Research, ${ }^{3}$ Department of Preventive Medicine, Yonsei University College of Medicine, Seoul, Republic of Korea*For correspondence: ecpark@yuhs.ac 
promoting behaviorsare beneficial to long-term cancer survivors so that interventions to promote these behaviors among cancer survivors can be developed.

\section{Materials and Methods}

We used data from the 2007 and 2012 Korea National Health and Nutrition Examination Surveys (KNHANES IV [2007 2009] and KNHANES V [2010 2012]). KNHANES IV and V are cross-sectional surveysthathavebeen conducted annually since 1998 by the Korean Centers for Disease Control and Prevention to assess the health and nutritional status of the Korean population. A stratified multi stage cluster sampling design was used to obtain a nationally representative sample. Each KNHANES surveyis composed of three parts: a health interview survey, a health examination, and a nutrition survey. We used data from the health interview survey component, which asked questions pertaining to socio-demographic characteristics, health status, medical history, healthcare utilization, quality of life (QOL), and behaviors, including cancer screening practices.

\section{Study sample}

Cancer survivors: The inclusion criteria were longterm cancer survivors; that is, individuals diagnosed with (stomach, liver, colorectal, breast, cervical, lung, thyroid, or other) cancer at least 5 years earlier.

Controls: Because of the exclusion of short-term cancer survivors, there could be a risk of selection bias. To solve this, general population controls were selected using propensity score matching (PSM). Logistic regressions generated propensity scores for the survivors and general population controls conditional upon the probability of the individual having had cancer and belonging to specific groups (age, sex, education, income, marital status, chronic disease status, depression, and region). A 5:1 ratio of controls to survivors was used. To confirm the balance between cancer survivors and the general population, chi-square tests were performed.

\section{Outcome measure: Health-promoting behaviors}

Smoking status, alcohol use, physical exercise, and disease screening were defined as health-promoting behaviors. These four self-reported variables were taken as dependent variables, and a multiple logistic regression was conducted for each dependent variable.

\section{Smoking status}

Participants who never smoked and ex-smokers were categorized as "Non-smokers", while those who smoked often or sometimes were categorized as "Smokers".

Table 1. General Characteristic by Cancer Status (Before and After PSM)

\begin{tabular}{|c|c|c|c|c|c|c|c|c|c|c|c|c|}
\hline & \multicolumn{6}{|c|}{ Before matching } & \multicolumn{6}{|c|}{ After matching } \\
\hline & \multirow[t]{3}{*}{ Total } & \multicolumn{2}{|c|}{$\begin{array}{l}\text { Long term } \\
\text { survival }\end{array}$} & \multicolumn{2}{|c|}{$\begin{array}{c}\text { General } \\
\text { population }\end{array}$} & \multirow[t]{3}{*}{$\mathrm{p}$ value } & \multirow[t]{2}{*}{ Total } & \multicolumn{2}{|c|}{$\begin{array}{l}\text { Long term } \\
\text { survival }\end{array}$} & \multicolumn{2}{|c|}{$\begin{array}{c}\text { General } \\
\text { population }\end{array}$} & \multirow[t]{3}{*}{$\mathrm{p}$ value } \\
\hline & & $\mathrm{N}$ & $\%$ & $\mathrm{~N}$ & $\%$ & & & $\mathrm{~N}$ & $\%$ & $\mathrm{~N}$ & $\%$ & \\
\hline & & 433 & 100 & 30721 & 100 & & & 433 & 100 & 2165 & 100 & \\
\hline \multicolumn{13}{|l|}{ Sex } \\
\hline Male & 13241 & 134 & 31.0 & 13107 & 42.7 & $<.0001$ & 804 & 134 & 31.0 & 670 & 31.0 & 1 \\
\hline Female & 17913 & 299 & 69.1 & 17614 & 57.3 & & 1794 & 299 & 69.1 & 1495 & 69.1 & \\
\hline \multicolumn{13}{|l|}{ Age } \\
\hline 49 or less & 15797 & 56 & 12.9 & 15741 & 51.2 & $<.0001$ & 336 & 56 & 12.9 & 280 & 12.9 & 1 \\
\hline $50-65$ & 8717 & 185 & 42.7 & 8532 & 27.8 & & 1110 & 185 & 42.7 & 925 & 42.7 & \\
\hline more than 65 & 6640 & 192 & 44.3 & 6448 & 21.0 & & 1152 & 192 & 44.3 & 960 & 44.3 & \\
\hline \multicolumn{13}{|l|}{ Education } \\
\hline College graduate & 8784 & 49 & 11.3 & 8735 & 28.4 & $<.0001$ & 339 & 49 & 11.3 & 290 & 13.4 & 0.3517 \\
\hline High school graduate & 10578 & 110 & 25.4 & 10468 & 34.1 & & 608 & 110 & 25.4 & 498 & 23.0 & \\
\hline Less than high school & 11792 & 274 & 63.3 & 11518 & 37.5 & & 1651 & 274 & 63.3 & 1377 & 63.6 & \\
\hline \multicolumn{13}{|l|}{ Income } \\
\hline Q4(High) & 8520 & 91 & 21.0 & 8429 & 27.4 & $<.0001$ & 519 & 91 & 21.0 & 428 & 19.8 & 0.9492 \\
\hline Q3 & 8437 & 89 & 20.6 & 8348 & 27.2 & & 543 & 89 & 20.6 & 454 & 21.0 & \\
\hline Q2 & 7885 & 108 & 24.9 & 7777 & 25.3 & & 658 & 108 & 24.9 & 550 & 25.4 & \\
\hline Q1(low) & 6312 & 145 & 33.5 & 6167 & 20.1 & & 878 & 145 & 33.5 & 733 & 33.9 & \\
\hline \multicolumn{13}{|l|}{ Marital status } \\
\hline Married & 26780 & 425 & 98.2 & 26355 & 85.8 & $<.0001$ & 2550 & 425 & 98.2 & 2125 & 98.2 & 1 \\
\hline Divorced, Widowed, Unmarried & 4374 & 8 & 1.9 & 4366 & 14.2 & & 48 & 8 & 1.9 & 40 & 1.9 & \\
\hline \multicolumn{13}{|l|}{ Chronic Disease } \\
\hline No & 22755 & 242 & 55.9 & 22513 & 73.3 & $<.0001$ & 1486 & 242 & 55.9 & 1244 & 57.5 & 0.5825 \\
\hline Yes & 8399 & 191 & 44.1 & 8208 & 26.7 & & 1112 & 191 & 44.1 & 921 & 42.5 & \\
\hline \multicolumn{13}{|l|}{ Depression } \\
\hline No & 26614 & 331 & 76.4 & 26283 & 85.6 & $<.0001$ & 1986 & 331 & 76.4 & 1655 & 76.4 & 1 \\
\hline Yes & 4540 & 102 & 23.6 & 4438 & 14.5 & & 612 & 102 & 23.6 & 510 & 23.6 & \\
\hline \multicolumn{13}{|l|}{ Region } \\
\hline Urban & 13930 & 199 & 46.0 & 13731 & 44.7 & 0.634 & 1075 & 199 & 46.0 & 876 & 40.5 & 0.0388 \\
\hline Rural & 17224 & 234 & 54.0 & 16990 & 55.3 & & 1523 & 234 & 54.0 & 1289 & 59.5 & \\
\hline Total & 31154 & 433 & 1.4 & 30721 & 98.6 & & 2598 & 433 & 16.7 & 2165 & 83.3 & \\
\hline
\end{tabular}




\section{Alcohol use}

Participants who never used alcohol or who consumed less than one glass of alcohol per month were as categorized as "Alcohol non-users", while those participants who consumed more than one glass of alcohol per month were categorized as "Alcohol users".

\section{Physical exercise}

Participants who reported that they did not participate in mild, moderate, or vigorous physical exercise at least once per week were categorized as "Non-exercisers", while those who engaged in any level of exercise more than once per week were categorized as "Regular exercisers".

\section{Disease screening}

Participants who did not undergo disease screening in the last 2 years were categorized as "Non-screening", while those who underwent screening in the last 2 years were categorized as "Screening".

\section{Independent variables}

In this study, the primary independent variable was cancer status. The subjects were divided into two groups, long-term cancer survivors and the general population, based on their cancer status.

The covariates considered in this study were age, sex, education, income, marital status, chronic disease status, depression, region and cancer type.

\section{Statistical analysis}

The chi-square test was used to evaluate statistical differences in characteristics and health-promoting behaviors between the survivors and general population controls. A multiple logistic regression model was applied to identify associations between cancer status (long-term cancer survivors vs.the general population) and health-promoting behaviors. All statistical analyses were performed by applying sampling weights for each individual, andp-values $<0.05$ were considered to indicate statistical significance. SAS 9.3 (SAS, Inc., Cary, NC, USA) was used for all analyses.

Table 3. Association between Status and Health Promoting Behavior

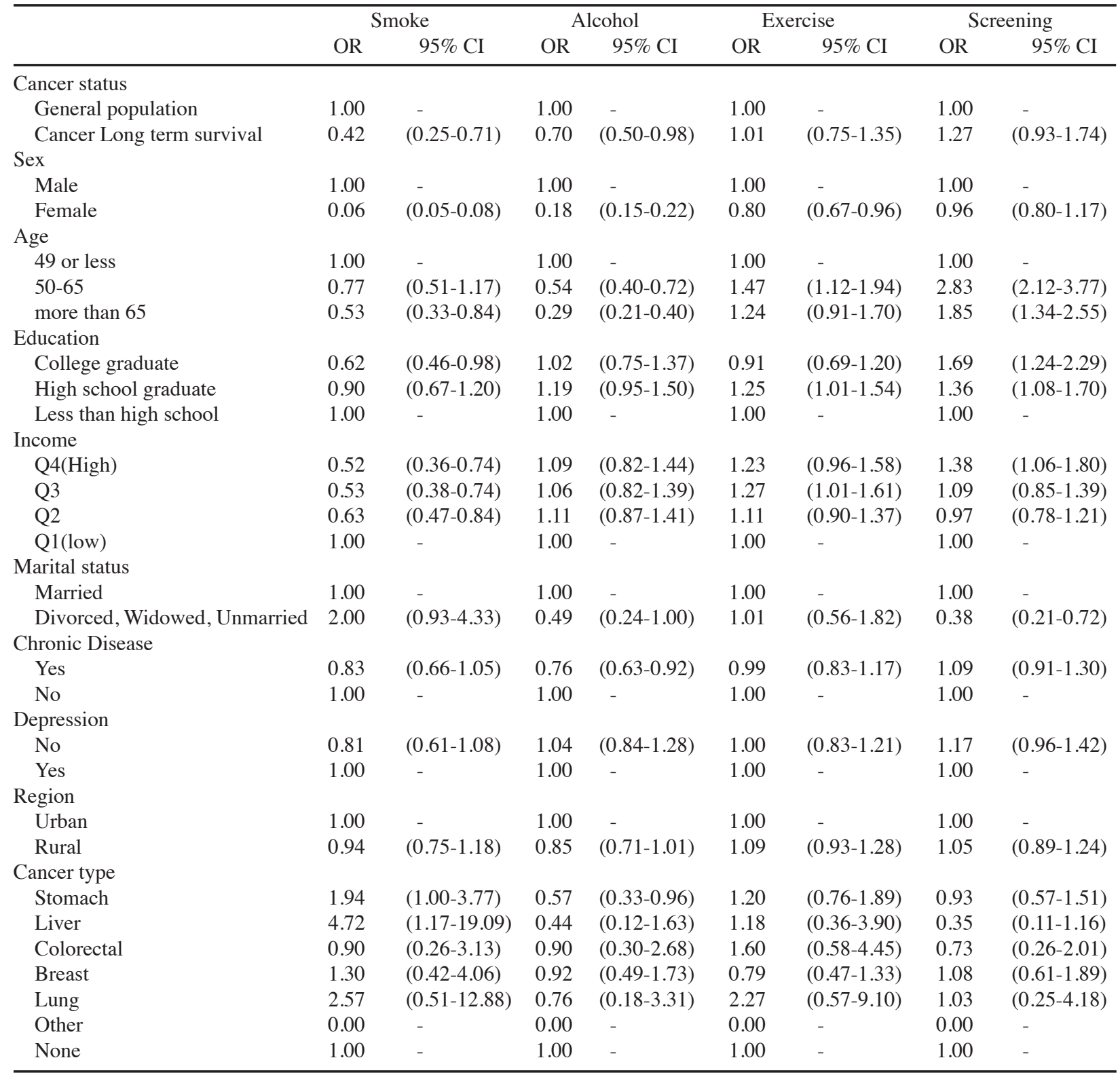




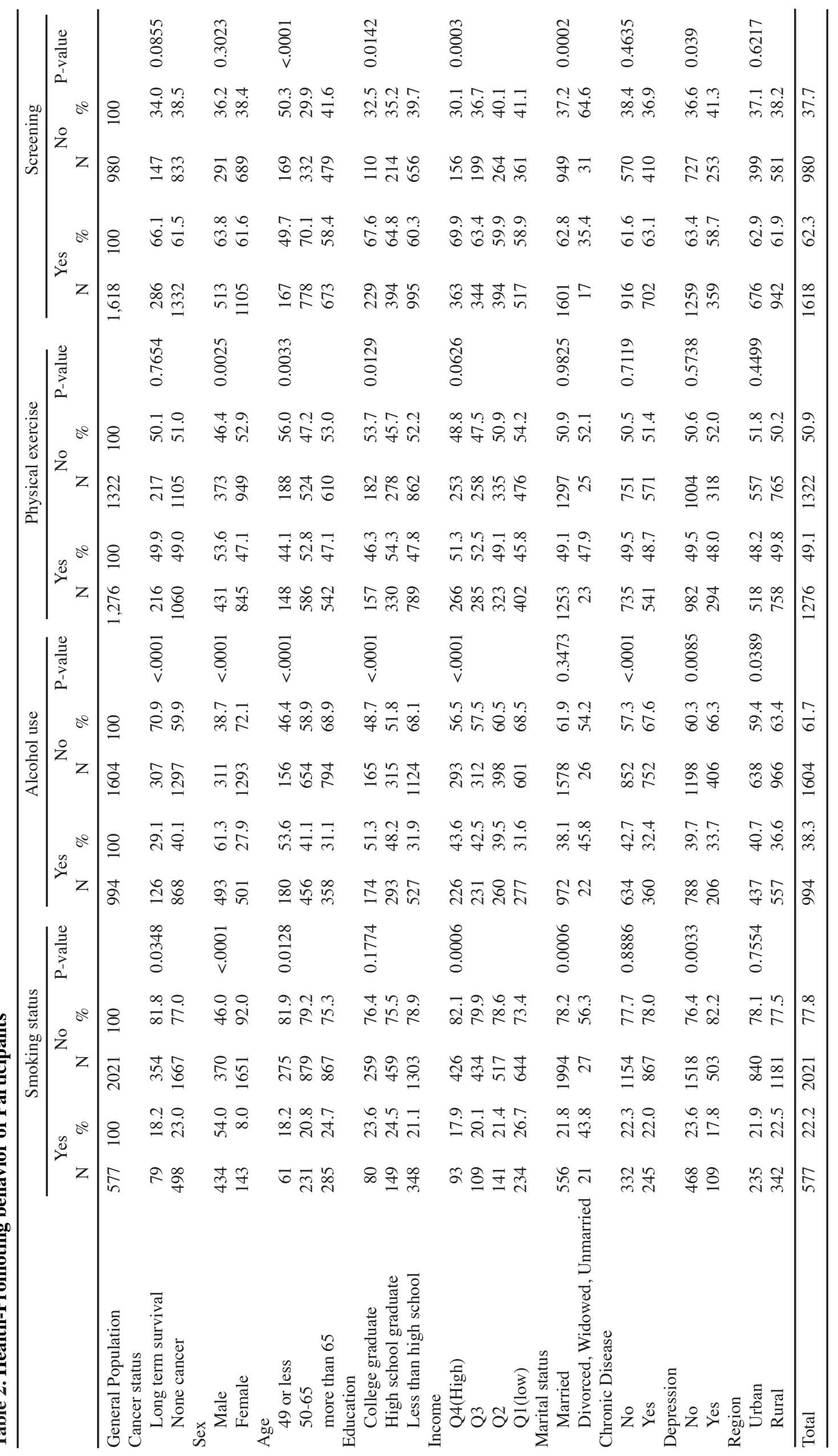

1418 Asian Pacific Journal of Cancer Prevention, Vol 16, 2015 


\section{Results}

Table 1 presents the general characteristics of the long-term cancer survivors and characteristics of the controls before and after PSM. Of 433 long-term cancer survivors, $69.1 \%$ were female and $31 \%$ were male. At lower education levels, the number of long-term cancer survivors increased. For comparing before and after PSM, chi-square tests were performed for the general population controls after PSM, and the general characteristics were found to be balanced between the long-term survivors and general population controls for almost all covariates.

Table 2 presents the frequency of positive healthpromoting behaviors by cancer status (long-term cancer survivors vs. general population controls). All positive health-promoting behaviors were more common among the long-term cancer survivors than the general population controls, but only smoking status and alcohol use was statistically significant by chi-square testing.

Table 3 presents the results of the multiple logistic regression analysis, which assessed the association between cancer status (long-term cancer survivors vs. general population controls) and health-promoting behaviors (smoking status, alcohol use, physical exercise, and disease screening). We calculated odds ratios (ORs) for the likelihood of smoking, likelihood of using alcohol, likelihood of doing physical exercise, and likelihood to undergo disease screening. Long-term cancer survivors had a lower risk of smoking than the general population controls (OR: 0.42, 95\% confidence interval [CI]: 0.250.71 ). In addition, the long-term cancer survivors had a lower risk of alcohol use than the general population controls (OR: 0.70, 95\%CI: 0.50-0.98). However, there was almost no difference in the likelihood of doing physical exercise or undergoing disease screening, and the difference that was found was not statistically significant (physical exercise OR: 1.01, 95\%CI: 0.75-1.35; disease screening OR: $1.27,95 \% \mathrm{CI}$ : 0.93-1.74).

\section{Discussion}

In this study, we assessed the association between cancer status (long-term cancer survivors vs. the general population) and health-promoting behaviors (smoking status, alcohol use, physical exercise, and disease screening). Our results indicate that long-term cancer survivors had a much lower risk of smoking and alcohol use than the general population controls (smoking OR: 0.42, 95\%CI: 0.25-0.71; alcohol use OR: $0.70,95 \% \mathrm{CI}$ : $0.50-0.98)$. On the other hand, almost no differences between the long-term cancer survivors and general population controls were identified for physical exercise and screening for cancer recurrence and secondary disease (physical exercise OR: 1.01, 95\%CI: 0.75-1.35; disease screening OR: $1.27,95 \%$ CI: 0.93-1.74). Previous studies support our findings. Several studies have found that cancer survivors exhibit more positive health-promoting behaviors in terms of smoking and alcohol use (Blanchard et al., 2008; Hawkins et al., 2010; Wang and Chung, 2012). Multiple studies have also found that cancer survivors do not get enough physical exercise or obtain screening for certain types of cancer (Trask et al., 2005; Mayer et al., 2007; Blanchard et al., 2008; Hudson et al., 2009; Schumacher et al., 2012; Low et al., 2014). In our study, physical exercise and disease screening were not significantly different between the long-term cancer survivors and general population controls. There are several possible reasons for this association. In terms of physical exercise, cancer survivors have greater difficulty getting enough exercise because of a decline in physical function. Survivors may find that treatment leaves them feeling unwell or without the energy to exercise, and even survivors who were not exercising before their diagnosis may experience declines in their overall level of household or occupational activity (Alfano et al., 2009; Cohen et al., 2012). In terms of disease screening, it could be that screening adherence varies by the type of cancer. Another reason might be a lack of recommendation by the patient's healthcare provider (Trask et al., 2005; Mayer et al., 2007). Greater intervention is needed in this area because of the benefit of health-promoting behaviors. Previous studies have found that positive health-promoting behaviors are helpful for the reasons described below.

For smoking and alcohol use, previous studies have shownthe harmful effects of continued smoking and alcohol use following a cancer diagnosis and during treatment. The harm may be immediate such as reduced treatment efficacy, increased side effects, and complications, but it can also take the form of increased rates of recurrence and second primary tumors, poorer overall survival, and decreased QOL (Wayne et al., 2008; Carmack et al., 2011; Bidstrup et al., 2013).

With regard to physical exercise, if cancer survivors are inactive it increases their risk of a second cancer, diabetes, cardiovascular disease, and disability. Also, it may lead to decreased cardiorespiratory fitness, bone loss, muscle atrophy, and worsening of glucose metabolism, insulin sensitivity, digestive function, and immune function (Morrow et al., 1999; Schmitz et al., 2005).

In terms of disease screening, screening can reduce the risk of dying from certain cancers through earlier detection when the stage of cancer is more amenable to effective treatment (Park et al., 2009; Khan et al., 2010; Schumacher et al., 2012).

Despite the increased health risks faced by the longterm cancer survivors in our study,most of them did not exhibit increased healthy lifestyle behaviors such as physical activity or screening for secondary cancers. Intervention is required to ensure that patients get physical exercise regularly, and that they undergo screening for secondary cancers and other diseasesbased on their healthcare provider's recommendations.

This study has a number of strengths. First, we used large-scale nationally representative data that were randomly sampled from the entire Korean population. Second, to avoid selection bias, we excluded short-term cancer survivors, and we matched the controls by PSM to minimize selection bias. We also analyzed each healthpromoting behavior separately using a multiple logistic regression model so that we could identify the risk of negative health-promoting behaviors after adjusting for a number of covariates. 
Despite the strengths mentioned above, this study also has some limitations. We used cross-sectional data, which preclude inferences regarding causal relationships. However, previous studies have found that cancer status changes an individual's health-related behavior. Thus, the consistency of our findings with these previous results could minimize the limitation. Additionally, a selfreported health-promoting behavior may be subject to recall bias. Finally, we were unable to obtain information about the stage of disease among the cancer patients or the severity of comorbidities.

In conclusion, the long-term cancer survivors had a much lower risk of smoking and alcohol use than the general population controls. In contrast, almost no differences between the long-term cancer survivors and general population controls were detected for physical exercise and screening for cancer recurrence or secondary diseases. To reduce the health risks and challenges facing cancer survivors, interventions should be developed that are aimed at increasing physical exercise and screening for cancer recurrence and secondary diseases.

\section{Acknowledgements}

This study was supported by a grant from the National R\&D Program for Cancer Control, Ministry of Health and Welfare, Republic of Korea (No. 1420230). The funding source had no role in the design and conduct of the study; collection, management, analysis, and interpretation of the data; or preparation, review, or approval of the manuscript. None of the authors have any conflicts of interest associated with this study.

\section{References}

Alfano CM, Day JM, Katz ML, et al (2009). Exercise and dietary change after diagnosis and cancer-related symptoms in long-term survivors of breast cancer: CALGB 79804. Psychooncology, 18, 128-33.

Bidstrup PE, Dalton SO, Christensen J, et al (2013). Changes in body mass index and alcohol and tobacco consumption among breast cancer survivors and cancer-free women: a prospective study in the danish diet, cancer and health cohort. Acta Oncol, 52, 327-35.

Blanchard CM, Courneya KS, Stein K, et al (2008). Cancer survivors' adherence to lifestyle behavior recommendations and associations with health-related quality of life: results from the American Cancer Society's SCS-II. J Clin Oncol, 26, 2198-204

Carmack CL, Basen-Engquist K, Gritz ER (2011). Survivors at higher risk for adverse late outcomes due to psychosocial and behavioral risk factors. Cancer Epidemiol Biomarkers Prev, 20, 2068-77.

Cohen HJ, Lan L, Archer L, et al (2012). Impact of age, comorbidity and symptoms on physical function in longterm breast cancer survivors (CALGB 70803). J Geriatr Oncol, 3, 82-9.

Hawkins NA, Smith T, Zhao L, et al (2010). Health-related behavior change after cancer: results of the American cancer society's studies of cancer survivors (SCS). J Cancer Surviv, 4, 20-32.

Hudson SV, Hahn KA, Ohman-Strickland P, et al (2009). Breast, colorectal and prostate cancer screening for cancer survivors and non-cancer patients in community practices. J Gen Intern Med, 24, 487-90.

Ihira H, Mizumoto A, Makino K, et al (2014). Physical functions, health-related outcomes, nutritional status, and blood markers in community-dwelling cancer survivors aged 75 years and older. Asian Pac J Cancer Prev, 15, 3305-10.

Khan NF, Carpenter L, Watson E, et al (2010). Cancer screening and preventative care among long-term cancer survivors in the United Kingdom. Br J Cancer, 102, 1085-90.

Lee JE, Loh SY (2013). Physical activity and quality of life of cancer survivors: a lack of focus for lifestyle redesign. Asian Pac J Cancer Prev, 14, 2551-5.

Low CA, Beckjord E, Bovbjerg DH, et al (2014). Correlates of positive health behaviors in cancer survivors: Results from the 2010 LIVESTRONG Survey. J Psychosoc Oncol, 32, 678-95.

Mayer DK, Terrin NC, Menon U, et al (2007). Screening practices in cancer survivors. J Cancer Surviv, 1, 17-26.

Morrow JR, Jr., Jackson AW, Bazzarre TL, et al (1999). A oneyear follow-up to physical activity and health. A report of the Surgeon General. Am J Prev Med, 17, 24-30.

Park SM, Park CT, Park SY, et al (2009). Factors related to second cancer screening practice in disease-free cervical cancer survivors. Cancer Causes Control, 20, 1697-703.

Schmitz KH, Holtzman J, Courneya KS, et al (2005). Controlled physical activity trials in cancer survivors: a systematic review and meta-analysis. Cancer Epidemiol Biomarkers Prev, 14, 1588-95.

Schumacher JR, Witt WP, Palta M, et al (2012). Cancer screening of long-term cancer survivors. J Am Board Fam Med, 25, 460-9.

Trask PC, Rabin C, Rogers ML, et al (2005). Cancer screening practices among cancer survivors. Am J Prev Med, 28, 351-6.

Wang HH, Chung UL (2012). Healthy lifestyle changes during the period before and after cancer diagnosis among breast cancer survivors. Asian Pac J Cancer Prev, 13, 4769-72.

Wayne S, Neuhouser ML, Ulrich CM, et al (2008). Association between alcohol intake and serum sex hormones and peptides differs by tamoxifen use in breast cancer survivors. Cancer Epidemiol Biomarkers Prev, 17, 3224-32. 